\title{
Sortie de conflit et capitalisme agraire en Colombie : une esquisse d'analyse
}

Jacobo Grajales

\section{OpenEdition}

\section{Journals}

Édition électronique

URL : https://journals.openedition.org/ideas/1844

DOI : 10.4000/ideas. 1844

ISSN : 1950-5701

Éditeur

Institut des Amériques

\section{Référence électronique}

Jacobo Grajales, « Sortie de conflit et capitalisme agraire en Colombie : une esquisse d'analyse », IdeAs [En ligne], 9 | 2017, mis en ligne le 03 juillet 2017, consulté le 18 octobre 2022. URL : http:// journals.openedition.org/ideas/1844; DOI : https://doi.org/10.4000/ideas.1844

Ce document a été généré automatiquement le 18 octobre 2022.

\section{(c) $(1)$}

Creative Commons - Attribution - Pas d'Utilisation Commerciale - Pas de Modification 4.0 International - CC BY-NC-ND 4.0

https://creativecommons.org/licenses/by-nc-nd/4.0/ 


\title{
Sortie de conflit et capitalisme agraire en Colombie : une esquisse d'analyse
}

\author{
Jacobo Grajales
}

1 Le vote de la loi sur les «zones d'intérêt pour le développement rural, économique et social » (ZIDRES) en janvier 2016, sa validation par la Cour constitutionnelle un an plus tard et la récente diffusion d'un projet de loi « d'aménagement social de la propriété et des terres rurales " témoignent d'une tension qui traverse la reconfiguration récente des politiques foncières et agraires en Colombie et qui sera probablement exacerbée par la mise en œuvre des accords de paix avec les FARC. Cette tension oppose deux conceptions du rapport entre politiques foncières et agraires et sortie de conflit.

2 Une première, défendue par les FARC, mais aussi par une partie de l'équipe de négociation qui a représenté l'État colombien à la Havane, voit dans ces politiques des vecteurs de pacification et de stabilisation. Dans une telle posture la terre rurale, ressource contentieuse placée au cœur du conflit armé, doit faire l'objet d'une redistribution guidée par des principes d'équité et de réparation. À cela s'ajoute la nécessité de rendre possibles des modèles économiques inclusifs, dont celui de l'agriculture familiale, porté par une partie du mouvement paysan colombien (Allain M. et Beuf A., 2014). Une telle politique économique et sociale rentrerait d'ailleurs dans le cadre d'une «réhabilitation» des zones marginales du pays, selon les termes d'un projet vieux d'au moins trois décennies.

3 Une seconde conception voit dans la stabilisation et la sécurisation - relatives - des campagnes colombiennes une opportunité pour faire du pays une puissance agricole régionale, à l'instar des «modèles" argentin et brésilien. La pacification du pays participerait ainsi à l'ouverture d'une "frontière agraire", rendant possible l'exploitation intensive de régions que la marginalité historique et l'emprise des guérillas ont transformées en zones sous-exploitées. Les plaines de l'Orénoque, mais aussi certaines vallées inter-andines et arrière-pays des littoraux sont intégrés dans un imaginaire « pionnier », fermement ancré dans l'histoire de la formation de l'État et du 
capitalisme colombien. Cette approche « développementaliste » de la " paix » n'est pas une simple réactivation de modèles anciens, mais interagit très directement avec un contexte économique international particulier. La succession récente de crises financière, alimentaire, énergétique - et les perspectives de renchérissement durable des matières premières ont effectivement donné lieu à un intérêt nouveau des investisseurs pour l'accès direct au contrôle sur la terre (Borras S., Hall R., Scoones I., White B., et al., 2011 ; Borras S. et Franco T., 2012 ; De Schutter O., 2011). En Colombie, comme partout dans les Suds, cela se traduit à la fois par une croissance des investissements directs étrangers dans l'agriculture, par une financiarisation des activités agro-industrielles et par un développement vertigineux des entreprises agroindustrielles nationales. Dans ce contexte, le "post-conflit» apparait comme une opportunité pour le développement.

La poursuite d'un tel modèle de développement est clairement contradictoire avec la refondation des relations de production autour du foncier rural que prônent les représentants des FARC et une grande partie du mouvement paysan colombien. Or, le modèle de la " paix libérale $~ "^{1}$, selon laquelle la sécurité permet une redynamisation du capitalisme agraire, est au cœur des négociations. Le principe fondamental énoncé à plusieurs reprises par le président Juan Manuel Santos selon lequel «le modèle économique ne se négocie pas » en est l'illustration la plus palpable.

Quelles sont les tensions générées par une telle contradiction? Quels sont les moyens que l'État met en œuvre pour les réduire et quels rapports de force s'ensuivent? Sans pouvoir approfondir ici ces questions ${ }^{2}$, ce texte posera quelques éléments généraux pour l'étude des recompositions du capitalisme agraire colombien dans la conjoncture actuelle.

6 La situation actuelle, qu'un bon nombre d'acteurs définissent dans les termes du postconflit, ne marque pas, si on l'observe du point de vue de l'économie politique du foncier rural, une rupture fondamentale. En effet, les dynamiques d'appropriation de la terre, de développement agro-industriel et de complémentarité entre la consolidation sécuritaire et le développement économique sont bien plus anciennes. Selon les territoires, elles peuvent se dater de la fin des années 1990 et du début des années 2000. D'autre part, les mobilisations qui réclament une protection des propriétés des déplacés forcés, une redistribution de la terre au nom de la pacification et une restitution des propriétés spoliées sont également déjà anciennes. Schématiquement, on peut considérer que l'on est face à deux processus parallèles.

7 Un premier processus correspond à une patrimonialisation, que l'on peut définir comme une "transformation de la terre en territoire» (Le Roy E., 2011) et qui met l'accent sur "l'encastrement social de la propriété », c'est-à-dire sur les relations sociales nouées autour de la terre et que celle-ci rend possibles (Polanyi K., 2009). Sans faire la socio-genèse d'une telle conception de la propriété en Colombie, on peut dater son intégration dans les textes juridiques et - de manière balbutiante - dans les politiques publiques du début des années 1990. La Constitution de 1991, la loi de 1993 sur les populations noires et celle de 1994 sur la «réforme agraire» en sont des exemples. Ces textes placent des limites à l'accumulation des terres, reconnaissent des formes d'auto-organisation paysanne comme les zones de réserve paysanne, et inaugurent une ethnicisation des territoires qui devient par la suite une dynamique puissante dans les conflits fonciers et plus largement dans la conflictualité socioenvironnementale (Bocarejo D., 2014). Ces dynamiques de patrimonialisation sont 
réactualisées par leur utilisation dans le cadre de mobilisations de résistance au déplacement forcé et à l'occupation du territoire par des acteurs armés. Les populations ethnicisées - noires et indigènes - sont particulièrement visibles lorsqu'elles font l'usage de l'idée de "territoire ", faisant appel aux protections légales que leur octroient la législation colombienne et les textes de droit international (Rolland S., 2014). Bien sûr, une telle ethnicisation des territoires est également porteuse de conflits entre des populations définies par des catégories ethniques et des populations paysannes dites "métisses" dont la protection est résiduelle (Ojeda D., 2012 ; Corredor J., 2014). De même, la visibilité accrue de certaines populations ne préjuge en rien du succès de leurs revendications, qui doivent souvent passer par le niveau transnational (la Cour interaméricaine des droits de l'homme notamment) pour avoir une quelconque efficacité.

Le second phénomène, que l'on peut qualifier de marchandisation, déjà esquissé plus haut, ne se réduit pas à une simple commercialisation accrue de la terre. Il correspond à une transformation de la terre en marchandise abstraite librement échangeable, donc à un «désencastrement social» de la propriété (Polanyi K., 2009). Bien sûr, cette dynamique est présente dès la formation initiale du capitalisme agraire - en Colombie comme ailleurs - et participe à des conflits fonciers très anciens. Or, elle prend des nouvelles formes à l'intérieur d'un paradigme de "paix libérale» qui relie explicitement la sécurisation du territoire et le développement capitaliste. Dès 2003, le gouvernement d'Álvaro Uribe cherchait à lever les maigres protections existantes sur les propriétés des déplacés afin d'allouer les "terres abandonnées" à des nouveaux producteurs ${ }^{3}$. À la même époque, les plaines de l'Orénoque sont progressivement redéfinies comme une nouvelle frontière agraire, ouverte à la "colonisation » agroindustrielle (Grajales J., 2016). Un travail technique et scientifique en vient à définir ce territoire - où l'on estime qu'il y a quatre millions d'hectares de terres aptes à l'agriculture - comme un espace vide et sous-utilisé. La recherche agronomique compare alors l'Orénoque colombien au Cerrado brésilien, une terre de savane que les conditions géologiques et pluviométriques rendent inapte à l'agriculture paysanne, mais que les techniques de l'agro-chimie moderne peuvent faire fleurir. Un modèle de développement basé sur la grande exploitation agro-industrielle et sur la privatisation des infrastructures de transport est mis sur pied. Cela correspond à une arrivée massive de nouveaux investisseurs, à la fois de grandes entreprises agro-industrielles colombiennes et étrangères et des petits et moyens acheteurs qui espèrent faire fortune grâce à l'augmentation du prix du foncier rural. D'autres territoires, comme les Montes de María étudiés par Ojeda et al. (2015), voient une dynamique similaire où la consolidation sécuritaire donne lieu à de nouvelles formes d'accaparement que la respectabilité légale rend plus solide que la spoliation paramilitaire des années récentes.

Dans ces conditions, le gouvernement colombien établit des équilibres précaires entre les pressions politiques contraires. D'une part, il a dû donner des gages aux FARC durant les négociations à la Havane sur sa volonté de conduire une réforme profonde des politiques foncières et agricoles. Il s'est appuyé pour cela sur des transformations institutionnelles qui étaient en cours depuis 2011, lorsque le gouvernement de Juan Manuel Santos fait de la politique de restitution de terres l'axe central de la réorientation symbolique de son action et de la renégociation de la place des agences de coopération étrangère dans le pays. Cette politique de restitution condense d'ailleurs une bonne partie des contradictions que l'on vient d'énoncer. Elle prend 
d'une part ses sources dans des pratiques de protection de la propriété des déplacés (Estrada M. et Rodríguez N., 2014), mais véhicule en même temps l'idée d'une formation d'un marché des terres libre et sécurisé, gage de développement économique et de confiance des investisseurs (Uprimny-Yepes R. et Sánchez N., 2010).

Les accords de paix participent d'ailleurs à ces équilibres précaires. Ainsi, l'État s'engage à soutenir la création de zones de réserve paysanne, une forme juridique longtemps bloquée par l'opposition des militaires qui y voyaient des espaces de refuge pour les FARC. En même temps, au lendemain du référendum gagné par les opposants aux accords, ceux-ci ont obtenu du gouvernement l'assurance que le soutien à l'agriculture paysanne ne porterait pas atteinte au modèle agro-industriel de développement.

11 Au fil des mobilisations, se profile ainsi une fragmentation des usages de la terre. Les territoires ethnicisés et les zones de réserve paysanne constitueraient des espaces de conflictualité qu'il conviendrait d'extraire du marché. Une certaine forme de "sanctuarisation" de ces espaces constituait l'une des conditions tacites d'une négociation avec les FARC. Or, la fragilité d'une telle situation est évidente. Les zones "patrimonialisées» ne le sont qu'en raison d'accords de circonstance, de la conjoncture politique et de la conflictualité récente. Des modèles alternatifs d'agriculture y sont légitimes mais seulement de manière résiduelle. Ces espaces sont délimités à l'intérieur d'un pays où la norme et l'idéal du développement est la production agro-industrielle. Il est indéniable que les FARC et les mouvements sociaux qui ont pris ancrage dans les zones rurales marginales sont profondément conscients du caractère précaire de leurs conquêtes récentes. C'est donc sur une ligne de crête entre marchandisation et patrimonialisation que les politiques foncières des années à venir redessineront les campagnes colombiennes.

\section{BIBLIOGRAPHIE}

Références

Allain Mathilde et Beuf Alice, «L'agriculture familiale et ses réappropriations locales par le mouvement paysan colombien », Revue Tiers Monde, 2014, vol. 4, n 4, p. 43-59.

Bocarejo Diana, « Legal Typologies and Topologies: The Construction of Indigenous Alterity and Its Spatialization Within the Colombian Constitutional Court ", Law \& Social Inquiry, 2014, vol. 39, $\mathrm{n}^{\circ} 2$, p. 334-360.

Borras Saturnino et Franco Jennifer, « Global land grabbing and trajectories of agrarian change: a preliminary analysis », Journal of Agrarian Change, 2012, vol. 12, n 1, p. 34-59.

Borras Saturnino M., Hall Ruth, Scoones Ian, White Ben, et al., « Towards a better understanding of global land grabbing: an editorial introduction », Journal of Peasant Studies, 2011, vol. 38, n 2, p. 209-216. 
Corredor Jessica, « Négociations identitaires et légitimités territoriales : les Chilapos dans le Bajo Atrato en Colombie », Cahiers de l'Urmis, 2014, nº 15.

De Schutter Olivier, « How not to think of land-grabbing: three critiques of large-scale investments in farmland », Journal of Peasant Studies, 2011, vol. 38, n 2, p. 249-279.

Estrada María del Rosario et Rodríguez Nadia Margarita, « La política de tierras para la población desplazada 2001-2011: de la protección a la restitución », Estudios Socio-Jurídicos, 2014, vol. 16, $\mathrm{n}^{\circ} 1$, p. $75-119$.

Grajales Jacobo, « De la spoliation à l'accumulation. Violence, pacification et conflits fonciers en Colombie », Critique Internationale, 2017, n 75, p. 21-36.

Grajales Jacobo, «La terre, entre guerre et paix. Politiques foncières et sortie de conflit en Colombie », Les Etudes du Ceri, 2016, n 223.

Le Roy Etienne, La terre de l'autre : Une anthropologie des régimes d'appropriation foncière, LGDJ, 2011.

Ojeda Diana, « Green pretexts: Ecotourism, neoliberal conservation and land grabbing in Tayrona National Natural Park, Colombia ", The Journal of Peasant Studies, 2012, vol. 39, n² 2, p. 357-375.

Ojeda Diana, Petzl Jennifer, Quiroga Catalina, Rodríguez Ana Catalina, et al., « Paisajes del despojo cotidiano: acaparamiento de tierra y agua en Montes de María, Colombia ", Revista de Estudios Sociales, 2015, n 35, p. 107-119.

Polanyi Karl, La Grande Transformation : Aux origines politiques et économiques de notre temps, Paris, Gallimard, 2009.

Pugh Michael C., Cooper Neil et Turner Mandy (dir.), Whose Peace?: Critical Perspectives on the Political Economy of Peacebuilding, Basingstoke England; New York, Palgrave Macmillan, 2008.

Rolland Stellio, « Pavarandó (Colombie), Des “communautés de paix" aux "zones humanitaires" ", Un monde de camps, M. Agier (dir.), Paris, La Découverte, 2014, p. 268-279.

Uprimny-Yepes Rodrigo et Sánchez Nelson Camilo, « Los dilemas de la restitución de tierras en Colombia », Estudios Socio-Jurídicos, 2010, vol. 12, n 2, p. 305-342.

\section{NOTES}

1. Pour une approche critique au débat de la "paix libérale » centrée sur l'économie politique de la sortie de guerre, voir (Pugh M., Cooper N. et Turner M., 2008)

2. Pour des développements plus approfondis voir (Grajales J., 2016, 2017).

3. Loi 812 de 2003. Plan nacional de desarrollo. Art. 28.

\section{AUTEUR}

JACOBO GRAJALES

Université de Lille, CERAPS 